\title{
Aminoácidos libres en líquido cefalorraquídeo de recién nacidos con encefalopatía hipóxico isquémica
}

\author{
Jorge Rodríguez O.'; Ximena Lecaros G. ${ }^{2}$; Magdalena Cortés S. $^{2}$ \\ Free aminoacids in the cerebrospinal fluid of \\ newborn infants with hipoxic ischemic encephalopathy
}

\begin{abstract}
Cerebrospinal fluid free arainoacid (AA) composition depends on blood - CSF barrier, nervous tissue and ependima coptalion. II has been previously reported that in mammalian cerebral cotlex a dectease of adenosinetriphosphate (ATP) is associated with an increase in asparlic acid, a deciease in glutamic acid, aminobulyric acid and glutamine contents and that fuel deprivolion causes changes in brain levels of ATP and those mentioned aminoacids. Using gas aromatography, ofter isobuthanol sterificalion of the carboxilic group and heptalluorbutyrate acylation of the amino group, free ominoocid conlent of cerebrospinal fluid and plasma were sludied in eighl normal control newborn infants and eight pallents with neonotal hipoxle ischemic encephalopathy in order to delecl changes in these late, but no differences ol aminoocid concentration in celebrospinal fluid were recorded omong bolh groups. This result is in centradiclion with recent reports of increased excitalory aminoacid iglutamic and aspartic ocid) concenirotion in the cerebrospinal fluid of six asphyxiated newborn infants, and of a good corselation of these chonges and neurologic prognosis of palients. More experience is needed in these resped.
\end{abstract}

(Koy words: amino ocids, cerebrospinal fluid cetebrol ischemia, newborn.)

Durante los primeros días del periodo neonatal, los aminoácidos libres (AA) del plasma experimentan cambios significativos, disminuyendo en general sus concentraciones ${ }^{1}$. La relación entre las concentraciones de los aminoácidos en el plasma y el líquido cefalorraquídeo (LCR) ha sido estudiada principalmente en edades más tardias $^{2-4}$ y para la mayoría de ellos es menor en el segundo caso, con coeficientes individuales de relación que sugieren que la composición aminoacídica de este último dependería fundamentalmente del paso a través de la barrera hematoencefálica, pero también de la captación por el tejido cerebral y ependimario de cada compuestos. Por otra parte, el glutamato y los aminoácidos relacionados, incluyendo glutamina aspartato y gama-aminobutotrato, se encuen-

1. Lniversidad de Valparaíso, Hospital Dr. Gustavo Fricke, Viña del Mar.

2. Bioquímico. Laboratorio Cromatografía Gascosa, Hospital Dr. Gustavo Fricke, Viña del Mar.

Trabajo favorecido con aportes del Proycclo 15/90, de la Dirección de Investigación Científica y Tecnológica de la Universidad de Valparaíso. tran en concentraciones elevadas en el parênquima cerebral, con respecto a otros tejidos ${ }^{6}$ y se ha sugerido que ellos juegan un importante rol como neurotransmisores ${ }^{7}$. Recientemente se ha señalado también que, en la corteza cerebral de mamíferos, la disminución de adenosina trifosfato (ATP) se asocia con aumento del aspartato y disminución del glutamato, aminobutotrato y glutamina y que la deprivación energética causa caídas significativas en los riveles de ATP de estos aminoácidos en el cerebro ${ }^{8}$.

La asfixia es una condición clínica en que la deprivación energética aguda está bien rcpresentada. Nuestro hipótesis de trabajo ha sido que pudiese existir un efecto directo de la asfixia sobre la difusión de aminócidos libres a través de la barrera hematoencefálica. El propósito de esta investigación fue medir cl contenido de aminoácidos en el plasma y el líquido cefalorraquídeo en recién nacidos con encefalopatía hipóxico isquémica y sin ella, con el objeto de comprobar si existen variaciones en csta situación con respecto a reciên nacidos sanos. 
Tabla

\section{Concontración de aminoácidos libres en líquido cefalorraquídeo de recién nacidos con y sin isquemia cerebral}

\begin{tabular}{|c|c|c|c|c|}
\hline \multirow{3}{*}{$\begin{array}{l}\text { Aninóácldos } \\
\mu o l / h\end{array}$} & \multicolumn{2}{|c|}{$\begin{array}{l}\text { Controles } \\
\text { (n: } 8)\end{array}$} & \multicolumn{2}{|c|}{$\begin{array}{l}\text { Enccfalopatía hipú- } \\
\text { xico-isquémica (n: } 8 \text { ) }\end{array}$} \\
\hline & 26 & $(0-190)$ & 29 & $(0-139)$ \\
\hline & 302 & $(12-1400)$ & 352 & $(0-1399)$ \\
\hline Glicina & 108 & $(19-306)$ & 152 & $(0-559)$ \\
\hline Valina & 206 & $(26-248)$ & 210 & $(18-270)$ \\
\hline Treonina & 187 & $(90-1200)$ & 219 & $(104-1314)$ \\
\hline Leucina & 14 & $(12-16)$ & 9 & $(2-14)$ \\
\hline Isoleucina & 58 & $(45-75)$ & 62 & $(9-356)$ \\
\hline Prolina & 299 & $(93-390)$ & 613 & $(18-720)$ \\
\hline Serina & 123 & $(80-148)$ & 79 & $(20-139)$ \\
\hline Aspargina & 70 & $(35-97)$ & 72 & $(40-92)$ \\
\hline Metionina & 27 & $(0-42)$ & 147 & $(18-216)$ \\
\hline Fenilalanina & 60 & $(52-69)$ & 43 & $(0-184)$ \\
\hline Omiúna & 6 & $(2-12)$ & 5 & $(0-9)$ \\
\hline Acido glutamático & 0.6 & $(0,2 \cdot 1,8)$ & 1.3 & $(0,3 \cdot 1,7)$ \\
\hline Lisina & 37 & $(20-49)$ & 30 & $(2-38)$ \\
\hline Acido aspártico & 6 & $(0-8)$ & 9 & $(0-25)$ \\
\hline 'Tirosina & 10 & $(8-18)$ & 8 & $(0-16)$ \\
\hline Triptofano & 1,3 & $(0-1,7)$ & 2,3 & $(0 \cdot 2,7)$ \\
\hline
\end{tabular}

Márgenes entre parćntcsis.

\section{Pacientes y Métodos}

Se estudiaron ocho tecién nacidos de término, cinco dc sexo fernenino, cuyo peso de nacimien to varió entre $2900 \mathrm{~g}$ y $3600 \mathrm{~g}$. En todos los pacientes la gestación había sido normal, el nacimiento por vía vaginal, la calificación de Apgar menor de 5 a los $5 \mathrm{~min}$. sufrieron depresión respiratoria atribuible a una situación aguda durante el parto, mos. traban síntomas clinicos clásicos de asfixia severa (coma, hipotonía y, en seis casos, convulsiones), hipoxemia y acidosis y cl diagnóstico de encefalopatía hipóxico isquémica en sus grados II o III fue hecho de acuerdo a los cricrios de SARNAT. Ninguno tenía signos de infección, trastomos electrolíticos. En sólo dos se registró modcrada hipoglicemia durante el período del estudio. En todos se registro, median te ultrasonografía en limpo real durante el período agudo de la encefalopatía hipóxico isquemica, disminueión va. riable pero significaliva de la pulsalilidad de arterias pericallosas y cpicorticales, asociada o no a hiperlucide $z$ de la sustancia blanca, to que fuc interpretado como consecuen. cia de hipoperfusión y edema cercbral. En ningún pacicnte se demostraion infartos isquémicos cerebrales o beucoma. lacia. No fueron incluidos recién nacidos con malnułrición intrauterina o sospecha de infección connatal, encefalopalía hipóxico isquémica con signos de hernorragias cercbrales periventriculares o parenquimalosas identificados mediante ultrasonido o en cuyos líquidos cerebroespinales se conta. ron más de cincuenta hematies por mm cúbico. Cinco de las recién nacidos seleccionsdos corro casos requinieron venilación mecánica durante el estudio.
En el electroenccíalograma de tres casos se registró un patrón de ondas lentas de supresión y en cinco la actividad eléctrica ccrebral mostzaba un patrón discontinuo de menos de tres oidas por segundo o actividad espicular. La actividad en cl suero de creatinafosfoquinasa (CPK) total eslaba moderadamente aumentada en tres pacientes en los cuales se deuentinó. Con una sola excepción los pacientes requirieton tratamiento con fenobarital en dosis usuales. Las punciones lumbares fueron realizadas entre las 48 y 72 horas de vida en los pacientes, en ausencia de nutrición parenteral con aminoácidos en el momento de obtener las muesiras, las que fueron recogidas en un tubo de vidno, después de haber dejado que $0.5 \mathrm{ml}$ del líquido hubiesen drenado a través de la aguja, enfriadas en hielo seco por un minuto y luego guardadas $a-20^{\circ} \mathrm{C}$ hasta su anślisis.

Los aminof́cidos fueron deteminados de acuerdo a un método previamente descrito". En síntesis, el líquido cefalorraquídeo de los pacientes fue centrifugado para remover rcslos celulares a $3000 \mathrm{rpm}$ durante $10 \mathrm{~min}$. Las muestras fucron deproteinizadas con ácido sulfosalicilico y se obtwvieron efluentes de los aminoácidos mediante columna con resina de intercambio ínico Dowex. Estos fueron sometidos a csterificación de su grupo carboxílico con isobutanol y ácido clorhídrico y acilación del grupo amino con anhúlído heptafluorbutírico y analizados mediante cromatografía ga seosa en un equipo "Shidmazu" (R) equipado con doble llama de hidrógeno en un programa lincal de temperatura, empleando nitrógeno corno gas transportador. Las columnas de vidnio fueron rellenadas con fase SUPELCO SE-30 al $3 \%$ y condicionadas $\perp 270^{\circ} \mathrm{C}$ durante 24 horas. La temperatura inicial de la columna durante la cromatografía fue de $100^{\circ} \mathrm{C}$ y la final $240^{\circ} \mathrm{C}$. La presión de salida del instnumento fue de $1.25 \mathrm{~kg} / \mathrm{cm}^{2}$.

La cantidad de amineácidos eluidos de la columna de cromalografía gaseosa se deteminó calculando la respuesla molar relativa a un esıándar interno de norleucina. La identificación de los picos se obtuvo mediante la derivalizaciôn individual de cada aminoácido y compatación posterior de los respectivos tiempos de retención. Lina segunda identificación de los picos fue realizada mediante coinyccción. [il área de los picos de aminoácidos fue calcu lada medisnte iniegración computacional realizada con un instrumento Cromalopac adosado al cromalógrafo ${ }^{\$}$. Los valores obcenidos fueron compazados con los registrados de ocho recién nacidos sanos de término y se expresaron como la media de los experimentos y sus desviaciones estândar. El significado fue calculado mediante el sistema STATPACK.

\section{Resultados}

La aminoaciduria fue normal en todos los pacientes y en dos casos las concentraciones plasmáticas de glicina presentó valores en los rangos límiles altos considerando la edad ${ }^{9}$.

Los tiempos de retención de los aminoácidos en el líquido cefalorraquideo variaron entre 6,38 y 21,03 minutos. La respuesta molar relativa a norleucina varió entre 0,6 y 1,99 . En la tigura se 


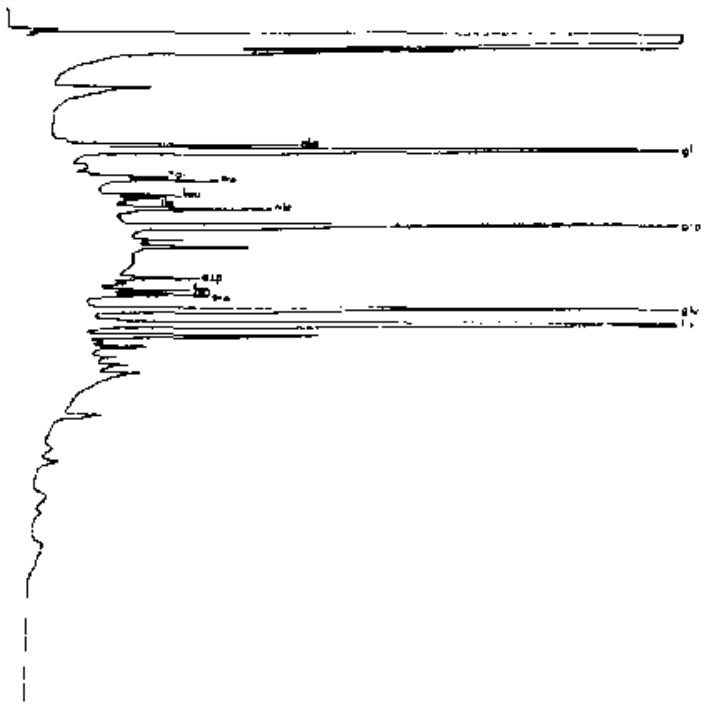

Figura: Cromatograma de los $N(O, S)$ - HBF - isobuti] esteres de aminoácidos en líquido cefalorraquídeo. Colum. na SE-30; 3\%, en un pacienle con encefalopalía hipóxico isquémica.

ilustra el cromatograma de aminoácidos libres en el líquido cerebroespinal de uno de los pacientes con encefalopatía hipóxico isquémica y en la tabla las concentraciones medias en recién nacidos con asfixia y controles con los márgenes de variación en dicho líquido. Los resultados en ambos grupos no mostraron diferencias significativas en el análisis estadístico.

\section{Comentario}

Los aminoácidos libres en líquido cefalorraquideo han sido estudiados con el objeto de definir su rol en la patogenia o en la expresión de diversas entidades clínicas. Según nuestros resultados no existiría diferencia en las concentraciones de aminoácidos libres del líquido cefalorraquídeo entre recién nacidos sanos y asfixiados. Recientemente se demostró que tampoco experimentan cambios significativos como consecuencia de convulsiones febriles ${ }^{10}$. En la esclerosis amiotrófica lateral se han encontrado elevadas las concentraciones de ácido glutámico en el plasna y líquido cefalorraquideo, sugiriento una anormalidad en el metabolismo del glutamato que podría estar relacionada con la patogenia de la enfermedad ${ }^{12}$.
La concentración de la mayoría del los aminoácidos libres en líquido cefalorraquídeo es consccuencia de transporte pasivo, desde el suero, por los plexos coró́deos. Sin embargo, algunos de ellos son Iransportados activamente a través de gradicntes de concentración, proceso que podría ser inhibido por escasez de la adenosina trifosfato necesaria para que se realicc. Nuestros resultados son consistentes con un mecanismo de transporte pasivo en la barrera hematoencefálica para la mayoria de los aminoácidos, es decir, no energético-dependiente y, en consecuencia, no afectado por la condición clínica de asfixia. La buena correlación que algunos han observado entre las concentraciones de ATP y la de determinados aminoácidos en la corteza cercbral -donde cl glutamato puede, incluso, ser metabolizado a aspartato a través del ciclo del citrato, a fin de producir ATP-, y que sugiere un mecanismo energético en la mantención de la concentración de estos aminoácidos, es en cierta forma contradictoria con estudios experimentales sobre el efecto del daño isquémico en las astas anteriores y posteriores de la sustancia gris en la médula de conejo, en los que no se encontraron cambios en las concentraciones de los aminoácidos libres, aspartato, glutamato y glicina, despućs de 10 min de isquemia seguidos por cuatro días de reperfusión ${ }^{12}$.

Nuestros resultados son también contradictorios con los descritos muy recientemente por autores finlandeses, que mostraban alteraciones en las concentraciones de aminoácidos en líquido cefalorraquídco de recién nacidos asfixiados, en comparación a los controles, cambios que afectaban particularmente a los ácidos aspártico y glutámico ${ }^{13}$. Desgraciadamente la muestra de recién nacidos con asfixia de ese estudio es aun más pequeña que la nuestra (6 pacientes) y la técnica empleada (HPLC) no nos permite aclarar la razón de esta diferencia de resultados. Parcce de extraordinaria significación la observación de esos investigadores, en el sentido que la mayor concentración de ácido aspártico se correlacionaría con peor pronóstico neurológico a largo píazo entre los niños asfixiados. Basados en los antecedentes recopilados de la literatura, antes de conocer la experiencia de Finlandia, csperábamos también encontrar cambios en las concentraciones de aminoácidos en el líquido cefalorraquídeo, que "nos permitiesen definir otro indicador biológico de asfixia, susceptible de ser 
correlacionado con el pronóstico neurológico, lo que no se cumplió. En nuestra opinión los aminoácidos libres en líquido cefalorraquídeo no representan un indicador seguro de compromiso neuronal en la asfixia, pero se requiere más experiencias antes de pronunciarse definitivamente sobre esta materia, en consideración al tamaño limitado de los grupos estudiados y lo contradictorio de los resultados.

\section{Resumen}

La composición de aminoácidos libres del líquido cefalonaquídeo depende del paso de éstos a través de la barrera hematoencefálica, de la síntesis en el tejido nervioso y de la captación ependimaria de aminoácidos. En la corteza cerebral de mamíferos, la disminución de la adenosina trifosfato (ATP) se encuentra asociada con aumento del aspartato y disminución de glutamato, aminobutirato y glutamina. Además, la deprivación energética modifica las concentraciones de ATP y de estos aminoácidos en el cerebro. Utilizando la técnica de cromatografía gaseosa asociada a derivatización, se estudió el contenido de aminoácidos libres en el líquido cefalorraquideo y plasma de ocho pacientes con encefalopatía hipóxico isquérnica, con el objeto de definir si existen variaciones con respecto al observado en recién nacidos no asfixiados. Las concentraciones medidas fueron similares en ambos grupos. Estos resultados son contradictorios con publicaciones recientes de autores finlandeses, que registraron aumento de las concentraciones de los aminoácidos exitatorios (glutamato y ácido aspártico) en el líquido cefalorraquídeo de seis recién nacidos asfixiados, el que guardaría relación con un mal pronóstico neurológico. En nuestra opinión se necesita mayor experiencia respecto a este problema.

(Palabras clave: isquemia cerebral, recién nacidos, aminoácidos, liquido cefalorraquídeo.)

\section{Agradecimientos}

A1 Dr. Raúl González A., la revisión crílica del manuscrito.

\section{Referencias}

1. Dickinson JC, Rosemblum H, and Hamitton PB: Ion exchange chromatography of the frec amino acids in the plasma of infants under 2500 grams at birth. Pediatrics 1970; 45: 606-613.

2. Perry $T L$, and Jones $R T$ : The amino acid content of human cerebrospinal fluid in nomal individuals and in mental defectives. I Clin Invest $1961 ; 40$; $1363-$ 1372.

3. Fisher RG, Pomeroy J, and Henry JP: The free amino acids in adult human cerebrosponal fluid. Acla Yeurol Scand 1968; 44: 619-630.

4. Dickinson $J C$, and Hamilton $P B$. The free amino acids of human spinal fluid determined by ion exchange chromalography. J Neurochem 1966: 3: 1179-1187.

5. Scriver $C H R$, and Rosemberg L: Metabolismo de los amioácidos y sus trastomos. In Vol IX Problemas actuales de la Pediatría Barcelona, 1979.

6. Lajtha AL, Maker HS, Clarke DD: Metabolism and transport of carbohydrates and amino acids. In: Siegel GJ, Albers RW, Agranoff BW, Kalzman RK, eds. Basic neurochemistry. 3rd ed. Boston: Little \& Brown, 1981 : 329-353.

7. Fonnum $F$ : Glubamale: a neurotransmitter in mammallian brain. J Neurochem 1984; 42: 1-11.

8. Ando $M$, Itoh $T$ : Changes of Amino Acid by the Deprivation of Energy Sources in the Cerebral Cortex. Brain Dev 1989: 11 : 169-174.

9. Rodriguez J. Pino $R$. Costa $E$ : Concentraciones de aminoácidos en sucro y orina en niños chilenos de $0-6$ años de edad. Rev Chil Pediatr 1987; 58: 436-441.

10. Cremades A, Penafiel $R$, Monserrat F, Cerbn $I$, and Perez-Flores D: Frec amino acids in the cerebrospinal cluid of children with febrite selzures. Neuropediatrics $1989 ; 20: 129 \cdot 131$.

11. Perry T, Krieger Ch, Jansen Sh, Etisen A: Amyotrophic lateral sclerosis: amino acid levels in plasma and ccrebrospinal fluid. Ann Neurol 1990; 28: 12.17.

12. Martinlok $J$, Saganaová $K$, and Chovko $M$ : Free and Peptide-Bound amino acids as indicators of ischemic damagc of the Rabbit Spinal Cord. J Neuropathol Exp Neurol 1991; 50: 73-81.

13. Rilkonen $R$, Kero $P$, Simell $O$ : Exilatory amino acids in cerebrospinal ftuid in neonatal asfixia. Pediatr Neurol 1992: $8: 37-40$. 